\title{
Tic en educación ¿problema- solución o posibilidad?
}

\section{Tic in education ¿problem-solution or possibility?}

\author{
Piedad Helena Betancur Zuluaga ${ }^{1}$
}

\begin{abstract}
Resumen
Gracias a los procesos de globalización, en los últimos años, las tecnologías irrumpieron en los campos laborales y educativos, alterando por completo las relaciones con la información y con el mundo. La escuela, el espacio de socialización por excelencia, era inevitable que estas llegaran a los espacios académicos, bajo la premisa de ser la solución a la dinámica y a los procesos de aprendizaje. Por lo anterior, el espacio educativo, debió repensarse a partir de este nuevo componente didáctico, que al mediar en el conocimiento, desvía el foco de atención sobre el docente y lo centra más en su mundo virtual. La educación, debe repensarse, dado que, existen voces, tanto a favor como en contra, que obligan a pensar una educación que, de manera equilibrada, optimice la máquina en función del saber, pero no descuide la formación humana de las nuevas generaciones.
\end{abstract}

Palabras clave: TIC; Tecnología; educación; pedagogía; comunicación; internet

\section{Abstract}

Thanks to the process of globalization, in the last few years, the technologies irrupted on the labor and educational fields, completely alternating the relationships not only with information but also with the

Licenciada en Educación Preescolar del Tecnológico de Antioquia. E-mail: helenabezu23@gmail.com 


\section{Piedad Helena Betancur Zuluaga}

world. In school, the socialization space for excellence, was inevitable that these were coming to the academic spaces, under the premise of being the solution to the dynamics and to the learning processes. By the previous thing, the educational space, should be had rethought from this new didactic component, which on having happened in the knowledge, turns the area of attention aside on the teacher and to center it more on his virtual world. The education must be rethought, given that, voices exist, so much as in favor as against, this forces to rethink an education that, in a balanced way, optimizes the machine depending on to know, but does not neglect the human formation of new generations.

Keywords: ICT; Technology; education; pedagogy; communication; internet.

\section{Un uso con diversas lecturas}

En la década de los años sesenta, el uso de las computadoras se empezó a popularizar en el espacio universitario y escolar de Estados Unidos. A medida que fue penetrando en la vida social y académica, sus aplicaciones se fueron adaptando, lo que permitió individualizar el proceso de aprendizaje, y terminó convirtiéndose en una herramienta útil para facilitar el acceso al conocimiento y servir de apoyo en los procesos de búsqueda y seguimiento a una temática específica con rapidez y seguridad.

El uso de las Tecnologías de la información y la comunicación en el medio escolar ha desatado toda suerte de polémicas, su implementación en la escuela, a través del aula informática, solo ha servido para que los estudiantes les den un uso más recreativo que académico. Por tanto, desde el punto de vista cognitivo, su impacto ha sido mínimo, puesto que aún no existe una disciplina de uso de los computadores y la escuela poco ha logrado que los estudiantes se conviertan en verdaderos investigadores y empiecen por tanto a optimizar una herramienta como el computador, para enriquecer su universo simbólico y conceptual.
Los desafíos en el espacio educativo tienen que ver, entonces, con la creación y optimización de verdaderas opciones de enseñanza que ofrezcan contenidos y propuestas de formación que den cuenta de las necesidades de los estudiantes y mantengan una disciplina tanto en el saber humanístico como en el técnico, puesto que la enseñanza, el conocimiento como saber universal y la pedagogía como tal, deben repensarse a partir de las nuevas posibilidades que ofrece la tecnología y de la manera como se presentan los contenidos curriculares.

Por lo anterior, habría que pensar que el rol del educador ( quien sirve de mediador) debe actualizarse en relación con las nuevas estrategias y opciones de aprendizaje que ofrecen las TIC, por tanto, los docentes que acompañan un proceso de formación, derivado de la tecnología, deben sobre todo saber gestionar los entornos virtuales y ser capaces de orientar y guiar a sus alumnos en el uso y aprovechamiento de la información, así como en las habilidades lectoras (icónico-verbal-sonoro), tanto de texto como de imagen, para potenciar en ellos verdaderas experiencias de aprendizaje, esto porque no podemos dejarnos llevar por la falsa creencia de que un alumno está muy preparado porque domina, de manera mecánica, el computador. Otras destrezas, sobre todo desde 


\section{Tic en educación ¿̇problema-solución o posibilidad?}

lo comunicacional, deben desarrollarse, como la de gestionar el conocimiento. De acuerdo con Salinas, el usuario en formación debe tener: (Salinas, 2002, pág. 98).

Acceso a un amplio rango de recursos de aprendizaje.

Control activo de los recursos de aprendizaje. Participación de los alumnos en experiencias de aprendizaje individualizadas, basadas en sus destrezas, conocimientos, intereses y objetivos. Acceso a grupos de aprendizaje colaborativo, que le permitan al alumno trabajar con otros para alcanzar objetivos en común.

Experiencias en tareas de resolución de problemas (o mejor, de resolución de dificultades emergentes antes que problemas preestablecidos) que son relevantes para los puestos de trabajo contemporáneos y futuros.

Es decir, una actitud frente al saber que saca al docente de su lugar de privilegio como fuente del saber y lo coloca como mediador, asesor y gestionador de los ambientes de aprendizaje. Un docente que logre interiorizar en sus estudiantes una serie de competencias que les permitan moverse con seguridad en el universo de la información, ser capaces de valorar datos, desechar la información inútil y derivar de todo ello un conocimiento para valerse por sí mismos en la sociedad moderna.

Es por esto, entonces, que la información, en el ámbito escolar, tiene un doble carácter: Como objeto de estudio y como medio, pues como lo dice María Irma Marabotto. "cuando una persona aprende la comprensión del significado implica muchas operaciones intelectuales que pueden sintetizarse muy rápidamente en dos grandes procesos: estrategias de elaboración de la información y estrategias de reducción de la información”. (Marabotto, 1999, p.166).

De ahí entonces que deba analizarse, de manera muy consciente, la responsabilidad que implica la enseńanza del uso y aprovechamiento de las tecnologías de la información, ya que deben tenerse en cuenta, por lo menos, alguno de los siguientes elementos. (Marabotto, 1999, p.166) Comprensión de la necesidad de tener conocimientos previos sobre el tema acerca del que se busca información para poder encontrarla. Comprensión de la necesidad de utilizar tecnologías para manipular información.

Comprensión del concepto de "palabra clave" y entrenamiento en su uso. Comprensión de las dificultades que implica la ambigüedad del lenguaje humano.

Toma de conciencia del problema de la sobreoferta de información y de la necesidad de seleccionar aquella útil y pertinente.

Es decir, una enseñanza que involucre aspectos éticos y humanos frente a la información como poder, como fin y como meta, puesto que hay que tener presente que las nuevas generaciones ya son reconocidas como "nativos digitales", con todo lo que ello implica, por lo que ya se les denomina "ciber-estudiantes", y otros términos similares como: Generación Net, Internet Generation, Generación Y, Millenials, Google Generation etc.

Se trata, por lo tanto, de saber integrar la tecnología al espacio educativo, adaptarla a los cambios del mundo y a las exigencias que la globalización supone a las nuevas generaciones. Es decir, no se puede desconocer el poder comunicativo que se desprende de las TIC y su incidencia no solo en el campo educativo sino también en el laboral, social, político y lo económico lo que obliga a repensar el conocimiento como una construcción multicultural. 
Por esta razón, como educadores, hay que ser capaces, de leer la realidad cultural, laboral y social a la que nos enfrentamos y que nos ubica frente al desafío de la desigualdad social y sus consecuencias, problema que puede enfrentarse, con una educación que forme en lo humano, lo solidario y lo ético a las nuevas generaciones nacidas en el tiempo que impera la tecnología. En el acto educativo intervienen diferentes variables que desbordan el acto de reconocer, leer y analizar información en una pantalla de computador, para convertirlo en un proceso más humano, más reflexivo y más interactivo con los sujetos. En las tecnologías hay un gran potencial de conocimiento, información y cultura que debe descubrirse, sin embargo, este proceso debe seguir siendo mediado por un pedagogo que posibilite el aprendizaje cooperativo, que potencie la creatividad, que favorezca las relaciones sociales y el desarrollo de nuevas habilidades comunicacionales afines con la nueva sociedad de la información, sociedad que, ha experimentado en los últimos ańos un avance tecnológico tan acelerado, tanto en información como en entretenimiento que todo incluso las relaciones y las comunicaciones, ha quedado supeditado a la rapidez.

Ahora bien, ¿qué puede esperarle a la educación tradicional frente a semejante avalancha informativa? La respuesta sigue siendo arriesgada, en cuanto estamos hablando desde los terrenos de la incertidumbre, desde una posición en la cual el conocimiento, como construcción lenta, producto de la lectura pausada y la reflexión, ya no parece tener sentido.

Tradicionalmente, la educación preparaba para la vida, formaba en unos saberes que conservaban un gran prestigio social y en ella la memoria era una habilidad prioritaria. Había un interés en formar un sujeto ético, intelectual y moralmente, que respondía a unos ideales de sociedad que a su vez estaban enmarcados en una escala de valores que brindaba cierta seguridad al entramado social.

Ahora el conocimiento es rápido, instantáneo, se ha convertido en una mercancía con valor de objeto de uso. El conocimiento se está revaluando constantemente y las nuevas generaciones no logran asimilarlo. Tendríamos, por tanto que pensar que la educación como espacio formador, debe reorganizarse como ente institucional, replantearse como lugar del saber (puesto que ya no lo es), y reconfigurarse en función de una nueva educación, que no pierda el horizonte ético, social, moral, intelectual y educativo que le corresponde.

En este sentido, no podemos dejar pasar las palabras de Zygmunt Bauman (2008) que continúan vigentes para todos:

En ningún otro punto de inflexión de la historia humana los educadores debieron afrontar un desafío estrictamente comparable con el que nos presenta la divisoria de aguas contemporáneas. Sencillamente, nunca antes estuvimos en una situación semejante. Aún debemos aprender el arte de vivir en un mundo sobresaturado de la información. Y también debemos aprender el aún más difícil arte de preparar a las próximas generaciones para vivir en semejante mundo. (Bauman, 2008, p.46).

Y para ese mundo futuro, debemos preparar a quienes hoy se encuentran en el espacio educativo. Esto porque la internet se ha convertido en el espacio de interacción, investigación y entretenimiento que más utilizan actualmente los jóvenes, es decir, es el medio multimedial por excelencia, para las nuevas maneras de acceder al conocimiento y entender cómo circulan los 
imaginarios culturales que están determinando a las nuevas generaciones puesto que la red multimedial: "crea sus propios lenguajes, sus propias formas de expresión (hablas), sus nuevas estéticas, nuevos imaginarios, nuevas percepciones de la realidad, pero también sus propias formas de ejercicio del poder". (Barrios,2009,p.270).

De acuerdo con lo anterior, habría que decir que la internet y todo el aparato tecnológico asociado a ella, se han convertido en la principal fuente de información y se han constituido en el espacio a través del cual la cultura global se socializa de manera más efectiva.

Es por esto que hoy se habla de una democratización de la cultura, es decir de una suerte de posibilidades para que todas las personas gocen de los mismos privilegios de conocer, entender y ver el mundo.

En este sentido, los conceptos de información y conocimiento han sufrido cambios desde el punto de vista de su significado inicial. Cuando se habla de información, esta se expresa desde los miles de millones de bytes que circulan en la red, pero cuyo acceso es permitido a quienes saben utilizar la tecnología. Por su parte el conocimiento está supeditado a las páginas especializadas que hacen parte del entorno digital.

Ahora bien, de acuerdo con Julio Cabero en: "Tecnología Educativa", la comunicación hace referencia a un "proceso que se desarrolla entre sujetos que disponen de algún tipo de convención y que mediante su utilización intercambian algo, indiferente de la situación espacio-temporal de cada uno de ellos". (Cabero, 1999, p.35).
Lo anterior nos remite, entonces, a una situación comunicativa en la cual se puede eliminar el espacio y por tanto la identidad entre los sujetos implicados y cuyo fundamento relacional se da de acuerdo con la habilidad para producir signos, construir códigos comunes y facilitar un intercambio (Cabero et al, 1999, p,37) "Un proceso de comunicación en el que no existe código y por consiguiente en el que no existe significación, queda reducido a un proceso de estímulo-respuesta”(Eco,1999,p,37).

En un mundo cada vez más mediatizado, conceptos como lectura y escritura han sido reelaborados para que hagan parte de los sistemas de hipertexto y en este sentido ya no hablamos de comunicación lineal sino de comunicación distribuida o modelo de redes. De acuerdo con esto, vale la pena repensar lo que al respecto plantea Alain Renaud:

Por lo tanto, las concepciones tradicionales sobre lectura, escritura, lenguaje, pensamiento espacio y tiempo, se están resquebrajando ante nuevas generaciones de seres humanos en las que el hipertexto vendría a reflejar la forma en que un nuevo pensamiento visual asocia datos e ideas, recoge información, pregunta causas y anticipa soluciones, gracias a un interfaz cuyos procesos y formatos de representación reforzarían una concepción operatoria de la inteligencia. La máquina informacional establece un puente entre lo que por mucho tiempo se pensó irreconciliable: El pensamiento técnico y el pensamiento simbólico. El instrumento ya no es una prolongación de la fuerza física sino una metáfora del cerebro" (Renaud, 1990, p, 11-26) citado por Moreno (1999).

De acuerdo con la anterior tanto el pensamiento técnico como el pensamiento simbólico ofrecen el verdadero desafío al aparato educativo 


\section{Piedad Helena Betancur Zuluaga}

tradicional, porque ¿̨cómo se potencia el primero y se enriquece el segundo? Esto nos lleva, por tanto, a centrar nuestra atención en generar nuevos modelos de enseńanza y aprendizaje, en optimizar los tiempos de interacción digital, en potenciar la construcción y la autonomía frente a la información, en favor del desarrollo de habilidades técnicas y la generación de estrategias cognitivas. Al respecto, Nicholas Negroponte (1995) propone tres interrogantes que, de acuerdo con su mirada, pueden dar respuesta a los cambios que se presentan en el aprendizaje con la llegada de las TIC (Moreno, 1999, p, 102).

¿Cómo aprenden los nińos?

¿Qué aprenden los niños?

¿Con quién aprenden los niños?

Estos tres interrogantes nos remiten necesariamente a los procesos de enseñanza y a la manera como se puede pedagogizar el conocimiento, apoyado en las nuevas tecnologías. Las TIC suponen un aprendiz autónomo, que puede explorar y controlar su propio aprendizaje, es decir, una posibilidad de desarrollar un interés cognitivo en los aprendices. Las TIC permiten también pensar en proyectos de trabajo colectivo, que pueden generar comunidades de conocimiento entre los estudiantes. En síntesis, se trata, entonces, de una escuela que puede facilitar los procesos de aprendizaje aprovechando las TIC.

Sentido de las TIC

De acuerdo con Tedesco (1995) "El nuevo pacto educativo", propone una descripción de las tres clases de efectos que las nuevas tecnologías de la información y la comunicación hacen sobre sus usuarios. Dichos efectos se catalogan así: alteran la estructura de intereses, cambian el carácter de los símbolos y modifican la naturaleza de la comunidad (Sancho, 2002, p, 31).
Es decir, una artificialidad de las relaciones humanas, que ya no necesitan de la interacción frente a frente, puesto que, estar conectado a través de las redes virtuales se convirtió en la manera de ser y estar con los otros. Por esta razón su uso en el espacio educativo exige metodologías adecuadas y estrategias apropiadas, para derivar de su utilización un verdadero complemento a la enseñanza tradicional. (Laurillard, 1993).

La idea del conocimiento académico como una visión abstracta platónica, como una forma abstracta platónica todavía no ha muerto, ha experimentado un nuevo ímpetu desde el desarrollo de modelos cognitivos de procesamiento de la información, que utilizan la metáfora de estructura del conocimiento o estructuras conceptuales para describir entidades mentalistas que puedan cambiarse por medio de instrucciones, o incluso representarse en programas de ordenador.

La manera de organizar, entender y retener el conocimiento, entonces, va a incidir de manera notable al momento de plantear nuevas situaciones de enseñanza-aprendizaje, ya que los educandos van a tener un nuevo punto de vista sobre un determinado tema, porque habrá que enseñar una manera de procesar el conocimiento para que se convierta en información útil y significativa y porque el papel de mediador de los docentes debe estar atravesado por una mentalidad abierta a la discusión, a que se ponga en tela de juicio su saber y a que se prioricen ciertos temas en función de los intereses personales de los estudiantes.

En el proceso de implementación de las TIC, a la vida escolar, se hace necesario revisar algunos elementos que, desde la sicología, enriquecen los microcurrículos de formación (contenidos pedagógicos). En este sentido, vale la pena mirar 
como punto de referencia las siguientes teorías: (González, Montoya y Urrego, 2000, p, 74): Aprendizaje verbal significativo y teorías de la asimilación: Enunciada por David Paul Ausubel. Teoría de la actividad: Formulada por la escuela de Lev Vygotsky.

Teoría de los esquemas de conocimiento: de Merril Anderson.

Teoría de la globalización: De M. Merril y Ch. Reigeluth.

Teoría del aprendizaje adulto.

Teoría genética: de Jean Piaget.

Las anteriores posturas teóricas engloban tanto los procesos de aprendizaje, como los enfoques que tienen que ver con la información y su proceso cognitivo que derivan en un saber. Buscan reestructurar los currículos, desde los que se forman a las jóvenes generaciones, no solo como usuarios de las nuevas tecnologías, sino también como ciudadanos responsables frente al manejo y la utilización que debe dársele a la información como proceso de aprendizaje para la vida social y laboral que dependerá, cada vez más, de procesos de producción mediados por las TIC.

En este orden de ideas, habría que pensar que, el aprendizaje debe volverse un acto significativo, en el cual el docente sea un verdadero experto en formular problemas, proponer iniciativas y permitir la interiorización de la autonomía y el aprendizaje colaborativo.

Por tanto, se retoman, las cuatro áreas temáticas que el proyecto CONEXIONES (2000) propone para la formación de un docente que quiere comprometerse con la mejora de la calidad de la educación, a través de la incorporación de las TIC a los procesos pedagógicos: área pedagógica, área didáctica, área tecnológica y área institucional (González, Montoya y Urrego, 2000, p 79).

Igualmente, se propone una quinta área temática, con el ánimo de contribuir a la reflexión sobre el impacto de las tecnologías en nuestros jóvenes y para pensarse como facilitadores del saber sin descuidar al sujeto en formación. Esa quinta área se denomina "ética del manejo o interacción" la cual comprende la enseńanza de unos patrones comportamentales frente al computador, que permitan optimizarlo como herramienta de trabajo y no como vehículo para perder el tiempo y llenarse de información banal, ya que la claridad de lo que se quiere y la conciencia de su búsqueda optimizarán mucho más los procesos de aprendizaje y de su interiorización.

Las nuevas tecnologías están clasificadas por tres tipos de generaciones que conviven con ellas en la sociedad actual. Las que cuentan con las habilidades innatas, para estos entornos digitales, las que se han tenido que adaptar a las tecnologías y las que se niegan a hacer parte de ellas.

Por lo anterior es importante comprender, como varía la forma en que la gente, las adopta y las concibe. Es decir que cada cual, desde sus niveles de identificación, puede terminar, por rechazar o por aceptar, la presencia de la tecnología, en su espacio social. Esto nos demuestra que no hay una línea definida a la que todos debamos adaptarnos, aunque en el fondo esta sea finalmente la intención, ya que también depende de unos imperativos culturales y de mercado, que lo que buscan es cambiar la vida y la forma de pensar, por medio del control que estos ejercen sobre nosotros.

Con la llegada de la red social "Facebook", el deseo natural de ser y estar en lo social, empieza a 
satisfacerse de manera inmediata, pues es en este estado que el ser humano se siente más persona, sin embargo, el problema es el progresivo aislamiento que la tecnología produce y que le puede llevar a convertirse en un ser insocial e insolidario.

¿Qué nos nivela en una misma categoría a conocidos y desconocidos?

La fascinación de ver y mostrar algo inocente y de agradecimiento a estas generosas publicaciones, ya que, generalmente desconocemos los peligros de esta sobreexposición, gracias a ella se empezaron a conocer: fraudes, robo de identidad, situaciones bochornosas. Se vulnera todo el tiempo la intimidad y se deja a un lado la verdadera relación de crecimiento mutuo, del contacto frente a frente, el despertar del pensamiento intuitivo, de poder mirarle al otro la sinceridad de sus gestos y reacciones para saber qué tanto puedo confiar en él. Precisamente lo que internet dificulta son las relaciones interpersonales, significativas o enriquecedoras.

Por tanto las redes sociales y sus usuarios se convirtieron en espacios de contactos donde los nexos se volvieron débiles, sin que estos demuestren una verdadera relación de amistad, cuando los motivos pueden ser por exposición y publicidad.

Con las tecnologías el lenguaje, se ha transformado demasiado rápido. Nuevas maneras de hablar, de interactuar y de expresión están generando otras formas culturales de establecer vínculos gracias a la inmediatez de las comunicaciones y a la sobreexposición informativa. Igualmente en otros ámbitos como la ciencia sus efectos han sido notorios.

Los mensajes que circulan por celular, internet, Facebook, Skype, Twitter, Messenger y correo electrónico, están simplificando el proceso escrito en una suerte de economía del lenguaje, que empobrece nuestra manera de usar la palabra en verdaderos procesos de reflexión. Lo que importa es calmar la ansiedad de comunicación, como si se tratara un trance para escapar de lo real.

La compulsión de este siglo parece ser la de estar conectado, sin ser consciente del propio aislamiento que nos sigue privando de la cercanía, el afecto y el encuentro que nos hacen personas. Al respecto, Baudrillard (1975), utiliza la "metáfora del espejo" para hablarnos de la telerealidad, a través de la cual "la televisión forma un referente de la realidad y lo transmite a la masa quien, por otro lado conforma su realidad [...] el contenido final que llega a la masa no es la realidad ni el referente, ni la interpretación sino un producto final que escenifica una realidad simulada y reinventada; la no-realidad" (Centenero y Frutos,2004, p,211).

Las TIC en el aula: miradas en contravía Entre finales del siglo XX y principios del siglo XXI, el auge y la expansión en el espacio social de las tecnologías de la información y la comunicación se han dado de una manera tan acelerada que, la vida de los países y la experiencia de la sociedad, a través de la interacción, se han visto alteradas por completo, pues se ha generado una comunidad que accede, cada vez más, a los espacios virtuales en busca de información o de posibilidades de entretenimiento.

Sin embargo este fenómeno de masas que logran entrar a un sinnúmero de contenidos de información no ha transformado los procesos de razonamiento, no ha logrado enriquecer los procesos cognitivos y no ha permitido formar sujetos con independencia de criterio; para poder referirnos a él como la verdadera revolución en el campo del saber. 
En los últimos años, las TIC, con todo su potencial tecnocrático, aparecen en el discurso educativo como una posibilidad de aprendizaje paralela a la escuela y como el espacio por excelencia para mejorar los niveles de comunicación. De ahí que se haya pensado que la integración de la tecnología a los procesos de enseńanza y aprendizaje iba a remediar para siempre los problemas cognitivos y los vacíos pedagógicos de los educandos.

Ya lo dijo Steve Jobs uno de los fundadores de la empresa informática APPLE: "Los problemas de la educación no pueden ser resueltos por la tecnología" (citado en Sancho, 2002). Las TIC, por tanto obedecen más a un proceso de globalización y es en este sentido que el papel mediador del docente debe encaminarse a la construcción de conocimiento válido para adaptarse al nuevo mundo y permitir a sus estudiantes una mejor asimilación del nuevo contexto cultural en el cual se desarrollará su vida.

Es por lo anterior, que se hace necesario, desde el espacio escolar, un nuevo proceso de alfabetización digital de los alumnos, mejores estrategias para enriquecer la productividad en general y la creación de un modelo pedagógico que logre articularse con las diversas herramientas informáticas, es decir, un trabajo de conjunto que logre aprovechar al máximo las nuevas posibilidades que ofrece la tecnología en el proceso educativo.

Esto supone un docente capaz de mantener un ambiente innovador en su metodología para lograr, de alguna manera, una escuela más eficaz e inclusiva, que logre adaptarse a las nuevas exigencias del mundo y rebajar los índices de fracaso escolar en las habilidades lingüísticas y matemáticas.
Habría que pensar que se requiere, por tanto un proceso de alfabetización digital que actualice y contextualice la educación, con las nuevas demandas surgidas del orden globalizado y orientadas a generar procesos productivos, aprovechando las ventajas que se derivan del espacio virtual, e innovar en las prácticas docentes de manera que el acto educativo se convierta en la verdadera posibilidad para enseñar un uso racional de las TIC.

Lo anterior porque, dada la velocidad de la información, las posibilidades infinitas de sorpresa que ofrece el mundo virtual y el bombardeo constante de publicidad e imágenes que configuran los gustos y estandarizan el deseo, hay que valorar el saber como una construcción personal que nos devuelva el sentido de lo humano, ya que:

"En el torbellino de cambios, el conocimiento se ajusta al uso instantáneo y se concibe para que se utilice una sola vez. Los conocimientos listos para el uso instantáneos e instantáneamente desechables de ese estilo que prometen los programas de software, resultan mucho más atractivos". (Bauman, 2008, p, 29-30).

En este sentido, se hace necesario pensar un proyecto educativo que involucre procesos de alfabetización digital y que logre integrar las áreas de conocimiento con una mirada transversal de la tecnología, puesto que las TIC exigen nuevas competencias para que la educación sea realmente significativa frente a los problemas y los desafíos de la vida contemporánea.

Este nuevo ambiente mediatizado se debe ampliar, así mismo el concepto de alfabetismo y desarrollar nuevos currículos y pedagogías que equilibren tanto la información derivada 
de los medios tecnológicos como de los medios impresos. "La tecnología, el multiculturalismo, las nuevas economías de la información y de los servicios, en el contexto de la globalización [...] están haciendo que la imagen sea más significativa que la escritura en muchos de los ámbitos de la comunicación pública”. (Snyder, 2002, p,68).

Las nuevas tecnologías, en la educación, siempre serán un desafío cognitivo para los educadores, pues su eficiencia dependerá, en gran parte, de la ética personal del docente (frente al saber y la comunicación) y de una interiorización por parte del alumno de unos patrones de conducta frente a las posibilidades de conocimiento a las que se enfrenta. En este orden de ideas, es valiosa la reflexión que hace Douglas M. Kellner al respecto:

Una teoría crítica de la tecnología es consciente de que las tecnologías no han previsto las consecuencias y que las buenas intenciones y los proyectos aparentemente buenos pueden tener resultados que no fueran deseados o positivos. En consecuencia, la cuestión no es si los ordenadores son buenos o malos en el aula o, más ampliamente, para la educación. Se trata más bien de una cuestión de qué hacer con ellos: ¿para qué propósitos útiles pueden servir los ordenadores? ¿qué clase de habilidades necesitan los estudiantes y los profesores para desplegar con efectividad los ordenadores y la tecnología de la información? ¿qué clase de efectos pueden tener los ordenadores y la tecnología de la información sobre el aprendizaje? ¿Qué nuevos alfabetismos, visiones de la educación y relaciones sociales necesitamos para democratizar y mejorar la educación hoy? (Snyder, 2002, p,231)

El uso educativo adecuado de las TIC tiene que ver con la conciencia del docente que sea capaz de darle sentido a la riqueza conceptual y metodológica que requiere la tecnología como componente de la educación, pues el conocimiento empieza a estar mediado por todo un universo de información que se desprende de todas las posibilidades icónicas, sonoras e interactivas, que desbordan la capacidad mental para procesarlas.

Ahora bien, el uso de los distintos tipos de aplicación informática, en el espacio educativo, debe permitirnos valorar sus posibilidades y recuperar la didáctica, con miras a un uso coherente y significativo, puesto que se trata de optimizar su riqueza informativa y la rapidez con la cual se logra acceder a ella, para educar, más en la utilización y contrastación del saber, que en el simple uso mecánico de reconocer diversas páginas virtuales.

\section{REFERENCIAS BIBLIOGRÁFICAS}

Barrios, A. ( 2009, enero-junio). Los Jóvenes y la red: Usos y consumos de los nuevos medios en la Sociedad de la información y la comunicación. Signo y Pensamiento, XXVIII(54).

Bauman, Z. (2008). Los Retos de la Educación en la Modernidad líquida. Barcelona: Gedisa. Cabero, J. (1999). El proceso Comunicativo en la enseńanza: Modelos Teóricos y elementos del proceso. En Cabero J. et al. Tecnología Educativa. Madrid: Síntesis.

Centenero, M. J. y Frutos, J, T. (2004). Cultura y simulacro, de Jean Baudrillard. (Reseña). Glosas Didácticas (12), 210-212) Barcelona: Kairós. Eco, U. (1999). El proceso Comunicativo en la Enseñanza: Modelos Teóricos y Elementos del proceso. En C. Julio y otros, Tecnología Educativa Madrid: Síntesis.

González, M., Montoya, J. y Urrego, I. (2000). Programa de formación de docentes: nuevas 
tecnologías y mejora de la calidad educativa. En González, M. Conexiones Medellín: Universidad Eafit; Universidad Pontificia Bolivariana.

Kellner M, D. (2002). Revolución Tecnológica, Alfabetismos Múltiples y la Reestructuración. En Snyder, llana Alfabetismos Digitales. Comunicación,innovavión y educación en ela era electrónica Málaga Aljibe.

Kress, G. (2002). Escritura de lo visual. En Snyder. Ilana, Alfabetismos Digitales. Comunicación,innovación y educación en la era eléctrónica. Malaga Aljibe.

Laurillard, D. (1993). Rethinking University teaching: a framework for effective use of educational technology.London: Routledge.

Marabotto, M. I. (1999). Información: La materia prima del siglo XXI. En Vera, R. y Asisten J.C. Yendo de la TIza al Mouse: Manual de informática educativa par docentes no informáticos (2 ed.). Buenos Aires, Argentina: Ediciones Novedades Educativas.

Martín Laborda, R. (2005). Cuadernos Sociedad de la Información. Las Nuevas Tecnologías en la Educación. Madrid: UNA.
Moreno, M. (1999). El aprendizaje, motor para la tríada más poderosa del siglo XXI. Información, comunicación y Tecnología. Signo y Pensamiento, 17(34), 89-104.

Negroponte, N. (1995). Ser digital (Vol. 17). Buenos Aires.

Rueda, R. (2007). Para una Pedagogía del Hipertexto. Una Teoría de la Deconstrucción y la Complejidad. Madrid: Anthropos.

Salinas, J. (2002, mayo-agosto). ¿Qué aportan las TIC a las Universidades Convencionales? Algunas Consideraciones y Reflexiones. Revista Educación y Pedagogía , XIV(33), 91-105.

Sancho, J. (2002, mayo-agosto). Las Tecnologías de la Información y la Comunicación en la Enseñanza Superior: Una aproximación Compleja Revista de Educación y Pedagogía, XIV(33), 31-48

Snyder, I. (2002). Alfabetismos digitales. Comunicación, innovación y educación en la era electrónica. Maloag: Aljibe. 